\title{
Mapeamento científico e tecnológico de Bauhinia L. (Fabaceae): Uma projeção para o potencial antimicrobiano
}

\author{
Scientific and technological mapping of Bauhinia L. (Fabaceae): A projection for antimicrobial \\ potential
}

Mapeo científico y tecnológico de Bauhinia L. (Fabaceae): una proyección del potencial antimicrobiano

Davi Nascimento Costa ORCID: https://orcid.org/0000-0002-1900-1754 Universidade Federal do Delta do Parnaíba, Brasil E-mail: daveoficial123@gmail.com

Ruanna Thaimires Brandão Souza ORCID: https://orcid.org/0000-0002-9416-8327 Universidade Federal do Piauí, Brasil E-mail: ruanna_na15@hotmail.com

Regigláucia Rodrigues de Oliveira ORCID: https://orcid.org/0000-0003-3916-3686 Universidade Federal do Piaú, Brasil E-mail: regiglaucia@hotmail.com

Renata Brito dos Reis

ORCID: https://orcid.org/0000-0003-4595-7810 Universidade Federal do Delta do Parnaíba, Brasil E-mail: renata_britoreis@hotmail.com

Maria Gracelia Paiva Nascimento ORCID: https://orcid.org/0000-0001-9652-5730 Universidade Federal do Piauí, Brasil E-mail: graceliapaiva@gmail.com

Giovanna Santos de Souza

ORCID: https://orcid.org/0000-0002-4982-0988 Universidade Federal do Piauí, Brasil

E-mail: gih.ssouza@gmail.com

Maria do Amparo de Moura Macêdo

ORCID: https://orcid.org/0000-0001-5176-0112 Universidade Federal do Piauí, Brasil

E-mail: amparo_macedo@hotmail.com

Nailton de Souza Araujo

ORCID: https://orcid.org/0000-0002-7529-5750 Universidade Federal do Piauí, Brasil

E-mail: nailtonbio4@gmail.com

Emerson Bruno Castro Mesquita

ORCID: https://orcid.org/0000-0003-0914-226X

Universidade Federal do Delta do Parnaíba, Brasil

E-mail: ebcmesquita@ hotmail.com

Graziela de Araújo Lima

ORCID: https://orcid.org/0000-0002-1833-0097 Universidade Estadual de Feira de Santana, Brasil

E-mail: grazilima17@hotmail.com

Ivanilza Moreira de Andrade

ORCID: https://orcid.org/0000-0001-6059-8540

Universidade Federal do Delta do Parnaíba, Brasil

E-mail: ivanilzaandrade@hotmail.com

\begin{abstract}
Resumo
Espécies de Bauhinia L. têm sido pesquisadas por apresentarem propriedades terapêuticas. Objetivou-se realizar um mapeamento tecnológico e científico envolvendo as atividades antimicrobianas de Bauhinia. Realizou-se uma prospecção científica e tecnológica em bases internacionais de dados de artigos e patentes, sobre as atividades antimicrobianas de Bauhinia. A Índia é o país que apresenta maior número de pesquisas que resultaram em publicações nas bases pesquisadas, com 28, 43 e 48 indexações nas bases Web of Science, Scopus e The Lens
\end{abstract}


respectivamente, seguida do Brasil e Malásia. As áreas com maior número de trabalhos indexados foram: Farmácia, Toxicologia e Farmacêutica (31,8\%). Os pedidos de depósitos de patentes iniciaram em $1984(\mathrm{n}=1)$ atingindo um limiar em 2004 ( $n=18)$. Nas bases pesquisadas os Estados Unidos da América são os maiores detentores de tecnologia entre os pedidos de depósitos $(\mathrm{n}=153)$. Com relação a CIP 48 patentes compreendem a subclasse A61K36. Conclui-se que o gênero é fonte de diversas pesquisas com ativos antimicrobianos e torna-se relevante a ampliação de produções tecnológicas e científicas com espécies do táxon.

Palavras-chave: Metabólitos secundários; Microbiologia; Plantas medicinais.

\begin{abstract}
Species of Bauhinia L. have been researched for presenting therapeutic properties. The objective was to perform a technological and scientific mapping involving the antimicrobial activities of Bauhinia. A scientific and technological prospection was carried out in international databases of articles and patents, about the antimicrobial activities of Bauhinia. India is the country with the highest number of researches that resulted in publications in the researched databases, with 28, 43 and 48 indexations in Web of Science, Scopus and The Lens, respectively, followed by Brazil and Malaysia. The areas with the highest number of indexed papers were: Pharmacy, Toxicology and Pharmaceutics (31.8\%). Patent applications started in $1984(n=1)$ reaching a threshold in $2004(n=18)$. In the searched databases the United States of America is the largest technology holder among the filing applications $(n=153)$. With respect to CIP 48 patents comprise subclass A61K36. It is concluded that the genus is a source of several researches with antimicrobial actives and it becomes relevant the expansion of technological and scientific productions with species of the taxon.
\end{abstract}

Keywords: Secondary metabolites; Microbiology; Medicinal plants.

\title{
Resumen
}

Las especies de Bauhinia L. han sido investigadas por presentar propiedades terapéuticas. El objetivo era realizar un mapeo tecnológico y científico de las actividades antimicrobianas de la Bauhinia. Se realizó una prospección científica y tecnológica en bases de datos internacionales de artículos y patentes sobre las actividades antimicrobianas de la Bauhinia. India es el país que presenta el mayor número de investigaciones que dieron lugar a publicaciones en las bases investigadas, con 28, 43 y 48 indexaciones en las bases Web of Science, Scopus y The Lens respectivamente, seguido de Brasil y Malasia. Las áreas con mayor número de trabajos indexados fueron: Farmacia, Toxicología y Farmacia (31,8\%). Las solicitudes de patentes se iniciaron en 1984 ( $\mathrm{n}=1)$ alcanzando un umbral en 2004 ( $n=18$ ). En las bases investigadas, los Estados Unidos de América son el mayor poseedor de tecnología entre las solicitudes presentadas $(\mathrm{n}=153)$. Con respecto al CIP 48 patentes comprenden la subclase A61K36. Se concluye que el género es fuente de diversas investigaciones con activos antimicrobianos y se hace relevante la ampliación de los productos tecnológicos y científicos con especies del táxon.

Palabras clave: Metabolitos secundarios. Microbiología. Plantas medicinales.

\section{Introdução}

O aumento da prevalência de infecções causadas por microrganismos resistentes aos antibióticos representa um dos maiores desafios para a Saúde Pública (Souza et al., 2021), pois prejudica avanços nos cuidados de saúde, produção de alimentos e expectativa de vida da população (Lima, Benjamim \& Santos, 2017).

As plantas representam uma fonte promissora de bioativos com potencial antimicrobiano os quais podem ser empregados na produção e síntese de um grande número de fármacos e fitoterápicos (Hasenclever et al., 2017).

Neste sentido, produtos naturais e compostos majoritários isolados de vegetais são investigados por sua capacidade de modular a resistência aos antibióticos no tratamento de doenças infecciosas causadas por organismos microbianos (Silva et al., 2020) e vêm despertando o interesse da indústria de medicamentos (Freitas et al., 2021).

Dentre os grupos de plantas pesquisados por suas propriedades terapêuticas e antimicrobianas, está o gênero Bauhinia L., pertencente à Fabaceae Lind., com 61 espécies para o Brasil, sendo 39 endêmicas, distribuídas na Mata Atlântica (11), Pantanal (4), Pampa (1), Amazônia (20), Caatinga (10) e a maior parte no cerrado (36) (Flora do Brasil 2020, 2021).

Espécies de Bauhinia vêm sendo utilizadas na medicina popular por apresentarem propriedades antioxidantes (Oliveira et al., 2020a), antimicrobianas (Lacerda et al., 2016), anti-inflamatória, hipoglicemiante e antitumorais (Chechinel Filho, 2009). No entanto, as pesquisas têm se intensificado principalmente por conta de seu uso contra o diabetes em comunidades tradicionais (Paula et al., 2014). 
Devido a rica diversidade e fácil acesso aos produtos naturais de origem vegetal, muitos países tem investido em pesquisas para obtenção de substâncias com atividade antimicrobianas advindas de plantas já conhecidas popularmente como medicinais. Podem ser citadas as espécies de Bauhinia L. (Bauhinia forficata Link. e Bauhinia ungulata L.) que já apresentaram atividade contra cepas bacterianas como Staphylococcus aureus (ATCC -American Type Culture Collection6538), Staphylococcus epidermidis (ATCC 12226), Micrococcus luteus (ATCC 9341), Escherichia coli (ATCC 8739), Klebsiella pneumoniae (ATCC 13883) e Pseudomonas aeruginosa (ATCC 25619) (Pereira et al., 2014).

Testes realizados por Farias et al. (2018), avaliaram a atividade antibacteriana do extrato foliar etanólico da espécie $B$. forficata L. frente as cepas de Staphylococcus aureus (ATCC 25923), Staphylococcus saprophyticus (ATCC 25352), Enterococcus faecalis (ATCC 29212), Escherichia coli (ATCC 25922), Pseudomonas aeruginosa (ATCC 27853) e Proteus mirabilis (ATCC 25933) evidenciaram atividades antibacterianas para as cepas $S$. aureus e E. faecalis.

Os efeitos biológicos e as atividades antimicrobianas de Bauhinia são atribuídos aos metabólitos secundários presentes em espécies do grupo, entre eles: taninos, terpenos, esteroides, ácidos aromáticos quinonas, lactonas, alcaloides e flavonoides (Oliveira et al., 2020b). Medeiros et al. (2016), caracterizaram a composição química do óleo essencial de $B$. ungulata e identificaram nove compostos majoritários, são eles: $\beta$-cariofileno, Óxido de cariofileno, $\alpha$-humuleno, epi-yeudesmol, $\alpha$-bisabolol, Copaeno, Nerolidol, $\alpha$-bisabol Óxido B e Espatulenol. Além disto, o óleo essencial apresentou atividade contra cepas bacterianas mostrando grande potencial antibacteriano.

Neste contexto, devido ao baixo acesso aos medicamentos industrializados pelas comunidades tradicionais, torna-se relevante validar a prática terapêutica alternativa, com o uso de plantas, objetivando a obtenção de efeitos medicamentosos, aplicação e uso contra agentes microbianos, o que agrega vantagens e pode contribuir com a diminuição de efeitos tóxicos e adversos decorrentes do uso de antibióticos e medicamentos utilizados no tratamento de patologias diversas (Silva et al., 2017).

Diante do exposto, objetivou-se realizar um mapeamento tecnológico e científico, a fim de traçar um histórico de produção de patentes e publicações científicas em bancos internacionais envolvendo as atividades antimicrobianas de Bauhinia L.

\section{Metodologia}

Esta pesquisa é do tipo documental, exploratória, de abordagem quantitativa (Gil, 2008), utilizando como descritores os termos: "Bauhinia AND antimicrobial", "Bauhinia AND antibacterial" e "Bauhinia AND antifungal", sendo considerados válidos os documentos que apresentassem esses termos no título e/ou resumo, publicados entre 1981 e 2021. A busca dos dados foi realizada em novembro de 2021.

Para realizar a prospecção tecnológica foram consultados bancos de patentes: World Intellectual Property Organizacion (WIPO), Derwent Innovation Index (DII), The Lens- Free \& Open Patent and Scholarly Search, sendo quantificados de acordo com a Classificação Internacional de Patente (CIP). Quando se realiza prospecção tecnológica, sabe-se que a CIP distribui as patentes de acordo com suas aplicabilidades. Sua estrutura de classificações segue uma ordem hierárquica dividida em oito seções, 21 subseções, 120 classes, 628 subclasses e 69.000 grupos (Serafini et al., 2012).

A prospecção científica foi obtida nas bases de artigos e resumos Web of Science, Scopus e The Lens- Free \& Open Patent and Scholarly Search e os documentos foram analisados quanto ao ano, países e áreas de publicação. A opção pela consulta nas respectivas bases de artigos e patentes se deu pela confiabilidade e versatilidade dos dados indexados principalmente para artigos científicos dado o elevado volume de publicações disponíveis.

Os dados foram tabulados de forma individual e organizados em tabelas e gráficos no Microsoft Excel (2019). A partir dos dados coletados, realizou-se o tratamento bibliométrico pela construção e análise de redes baseadas na bibliografia selecionada a partir do Software VOSview versão 1.6.15 (Van Eck \& Waltman, 2010), cuja ênfase está na análise e na 
visualização de agrupamentos de dados bibliográficos utilizando a abordagem distance-based. A base selecionada para análise foi a Scopus.

De modo amplo, os nodos da rede bibliográfica são analisados de maneira que a distância entre eles indica aproximadamente sua relação, segundo determinados critérios de agregação, conformando um mapa (Palludeto \& Filipini, 2019). Neste estudo, utilizou-se o critério de co-ocorrência (de termos relevantes) que procura identificar elementos comuns aos textos da base de dados e termos significativos que estejam interligados.

\section{Resultados e Discussão}

Utilizando as bases de artigos Scopus, Web of Science e The Lens, com emprego dos descritores "Bauhinia AND Antimicrobial", obteve-se o total de 110, 58, 344 artigos indexados, respectivamente. No entanto, com o objetivo de refinar os dados realizou-se uma busca com os descritores "Bauhinia AND Antibacterial" e a quantidade de trabalhos indexados correspondeu a 106 (Scopus), 56 (Web of Science) e 304 (The Lens). Já empregando os descritores "Bauhinia AND Antifungal” registrou-se 40, 55 e 163 pesquisas indexadas nas bases Scopus, Web of Science e The Lens, respectivamente.

Para a busca de tecnologias, foram registradas para os descritores "Bauhinia AND Antimicrobial” um, 20 e 218 patentes, nesta ordem na WIPO, DII e The Lens, enquanto para o emprego dos termos "Bauhinia AND Antibacterial” foram encontradas nove (WIPO), 43 (DII) e 100 (The Lens) tecnologias indexadas. Para os descritores "Bauhinia AND Antifungal" foram registradas 113 (WIPO) e 163 (The Lens) patentes e nenhum registro para a base DII (Tabela 1).

Tabela 1. Número de artigos e patentes registrados por bases de dados com os descritores "Bauhinia” AND "Antimicrobial", "Bauhinia AND Antibacterial” e "Bauhinia AND Antifungal”.

\begin{tabular}{ccccccc}
\hline Descritores & Scopus & Web of Science & The Lens & WIPO & DII & The Lens \\
\hline Bauhinia AND Antimicrobial & 110 & 58 & 344 & 1 & 20 & 218 \\
Bauhinia AND Antibacterial & 106 & 56 & 304 & 9 & 43 & 100 \\
Bauhinia AND Antifungal & 40 & 55 & 163 & 113 & 0 & 163 \\
\hline Total & $\mathbf{2 5 6}$ & $\mathbf{1 6 9}$ & $\mathbf{8 1 1}$ & $\mathbf{1 2 3}$ & $\mathbf{6 3}$ & $\mathbf{4 8 1}$ \\
\hline
\end{tabular}

Fonte: Autores (2021).

\subsection{Mapeamento tecnológico}

A partir da busca com os descritores selecionados na The Lens, DII e WIPO, observou-se que os depósitos iniciaram em 1984 (Figura 1) com limiar de tecnologias em 2004 ( $\mathrm{n=18)}$. De acordo com os dados da The Lens (2021), no início dos anos 2000 até 2004, os depósitos tiveram como principal foco a prevenção e tratamento de desordens intestinais, isto se justifica pelo aumento da resistência a antibióticos utilizados no tratamento de doenças gastrointestinais e transmitidas por alimentos (Nadvorny, Figueiredo \& Schmidt, 2004; Mendes et al., 2004).

Além disto, analisa-se também que na última década houve um aumento na indexação de patentes. A química de produtos naturais vem representando um papel importante na obtenção de substâncias bioativas e no desenvolvimento de novos fármacos no setor industrial nos últimos anos, principalmente, devido ao aumento da resistência bacteriana e surgimento de cepas resistentes aos antibióticos já existentes (Silva et al., 2020). 
Figura 1. Evolução anual dos depósitos de patente com os termos "Bauhinia AND Antimicrobial" nas bases de patentes The Lens, Derwent Innovation Index (DII) e World Intellectual Property Organizacion (WIPO).

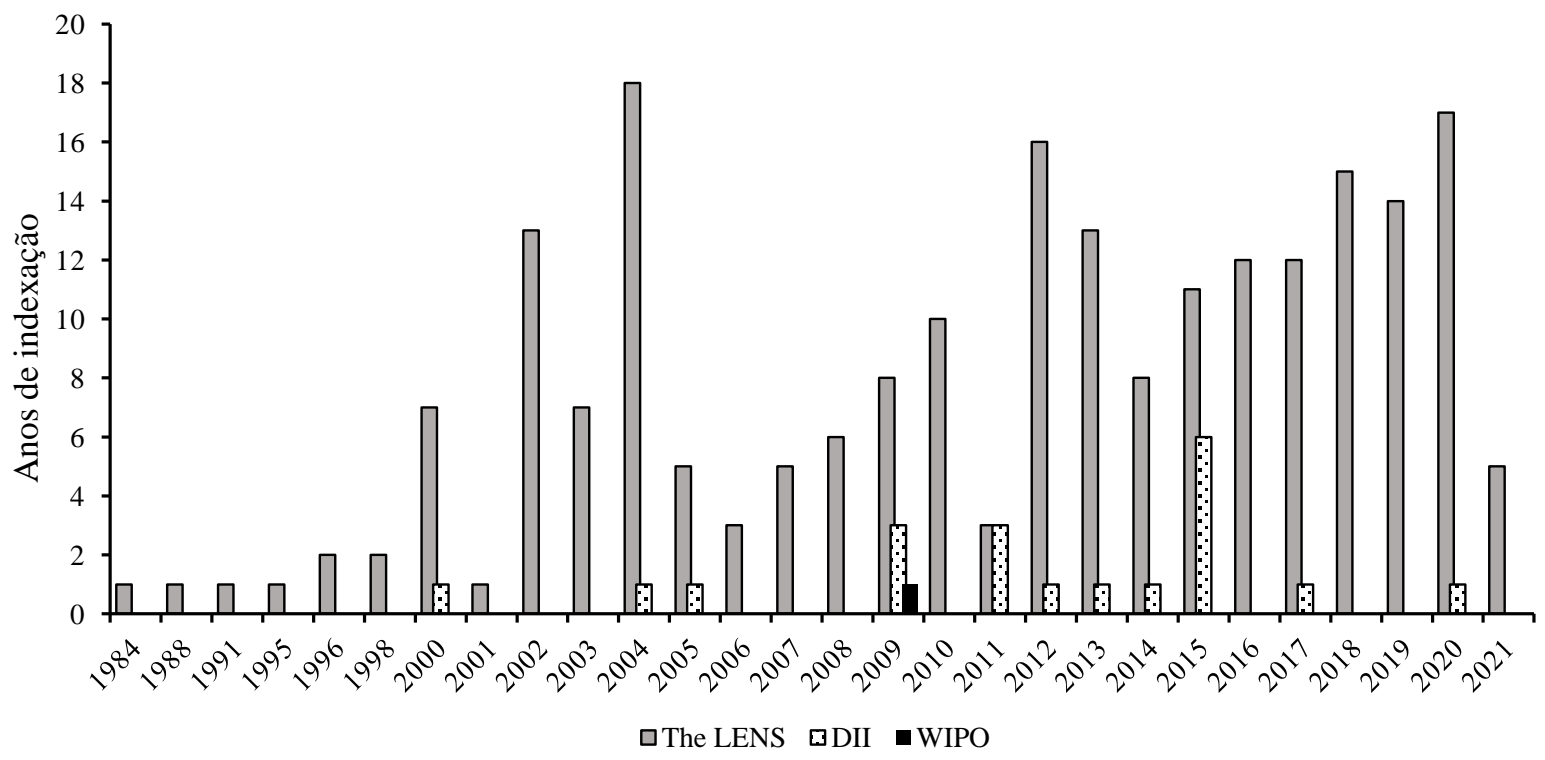

Fonte: Autores (2021).

Analisando a CIP das patentes depositadas, verificou-se que todas correspondem à Seção A (Necessidade Humanas) (WIPO, 2021). Entre os pedidos de depósitos de tecnologias nas bases internacionais 48 estão alocados na subclasse A61K36 (Figura 2), que trata de preparações medicinais contendo materiais de constituição indeterminada derivada de algas, líquens, fungos ou plantas, ou derivados dos mesmos, por exemplo medicamentos tradicionais à base de ervas. Um número considerável de patentes está inserido na subclasse A61K8 (48), relacionada a Cosméticos e preparações de banheiros similares; e na subclasse A61P35 (47), que relaciona agentes antineoplásicos (classe de fármacos utilizados para diminuir células tumorais). Outras patentes em menor número estão inseridas nas subclasses A61K38 (Preparações medicinais contendo peptídeos), A61K20 (Pílulas, lonzenges ou comprimidos) e A61K39 (Preparações medicinais contendo antígenos ou anticorpos).

Figura 2. Distribuição por CIP dos depósitos de patentes com o termo "Bauhinia AND Antimicrobial" nas bases The Lens, Derwent Innovation Index (DII) e World Intellectual Property Organizacion (WIPO).

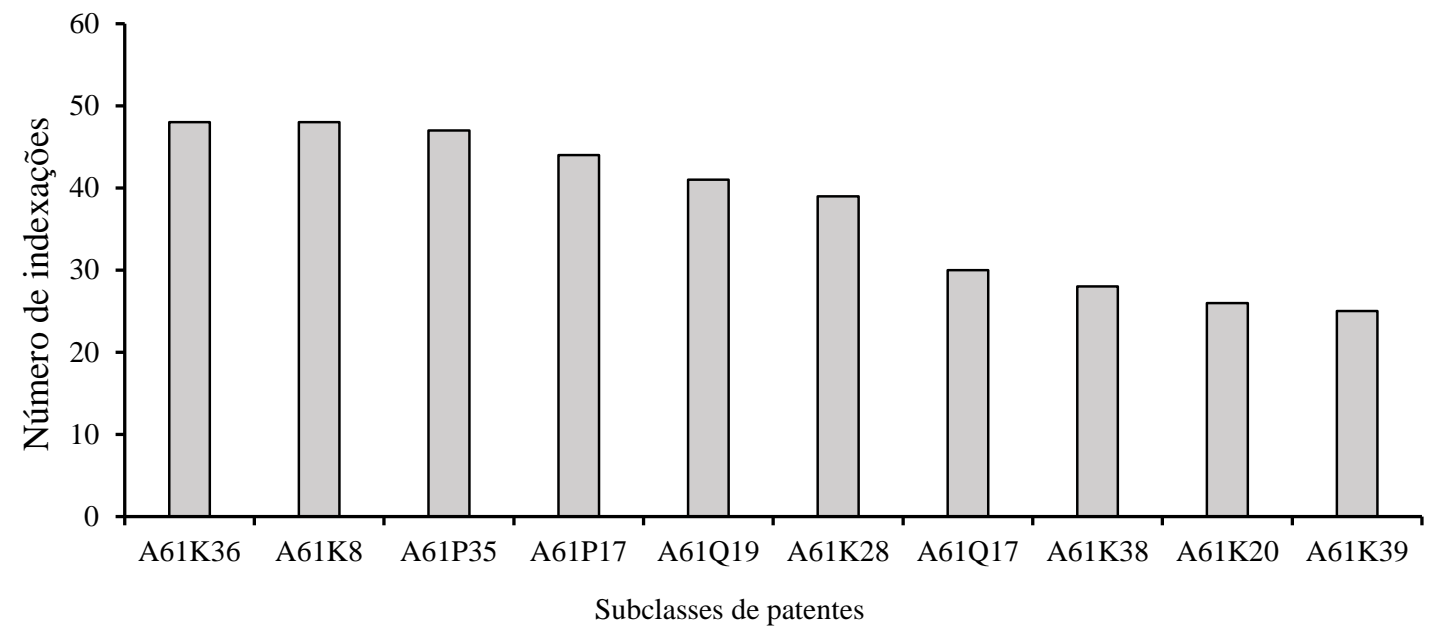

Fonte: Autores (2021). 
De acordo com os dados levantados durante a pesquisa os Estados Unidos da América (EUA) foi o país com maior número de depósitos de patentes ( $\mathrm{n}=153$ ). Esse país foi o pioneiro no desenvolvimento da Biotecnologia e permanece como um dos líderes neste campo. A influência Norte-americana em pesquisas abrange de forma significativa as áreas relacionadas à Genética vegetal, Medicina e Atividade antimicrobiana (Fontes, 2019).

O Relatório de ameaças de resistência a antibióticos do Centers for Disease Control and Prevention- CDC (Centro de Controle e Prevenção de Doenças), apontou que o número de infecções por patógenos microbianos é elevado, de acordo com a estimativa mais de 35.000 pessoas vêm a óbito anualmente, por causa da resistência aos antibióticos, o que reforça a busca dos Norte-americanos por tratamentos alternativos (CDC, 2019).

De acordo com a WIPO (2021), a única patente depositada com os descritores "Bauhinia AND Antimicrobial" relaciona-se ao uso de inibidores de protease isolados de Bauhinia sp. para o tratamento de infecções microbianas e composição farmacêutica. A tecnologia foi patenteada por pesquisadores brasileiros em 2009.

\subsection{Mapeamento científico}

A Índia é o país que apresenta maior número de pesquisas que resultaram em publicações nas bases pesquisadas, apresentando 28, 43 e 48 indexações nas bases Web of Science, Scopus e The Lens, respectivamente (Figura 3), Brasil (WoS= 12, Scopus $=29$, e The Lens $=44$ ) e Malásia (Scopus= 5, WoS $=6$ e The Lens=12). África do Sul e Egito apresentam menor número de publicações associando o táxon as atividades antimicrobianas. Alguns países em desenvolvimento, como por exemplo, a Índia vem ocupando espaços relevantes nas pesquisas e desenvolvimento de tecnologia na indústria farmacêutica. Nas últimas quatro décadas, o país vem acirrando o debate sobre a utilização e produção de fármacos naturais que possam ser incorporados e assimilados em âmbito local (Radaelli \& Paranhos, 2015).

Figura 3. Distribuição por países de artigos indexados nas bases Scopus, The Lens e Web of Science com os termos "Bauhinia AND Antimicrobial".

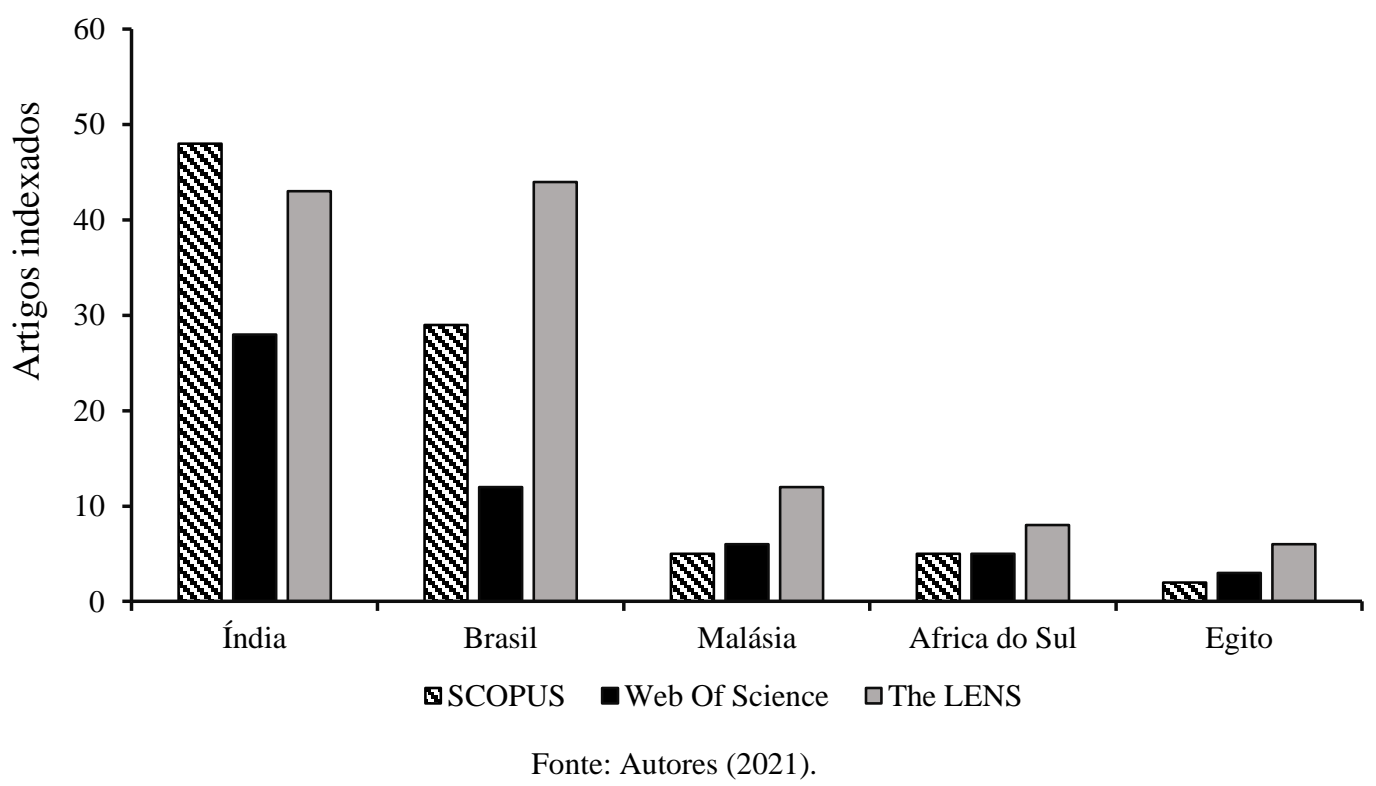

O Brasil é detentor da maior diversidade biológica do mundo, bem como um vasto conhecimento tradicional frente a diversidade étnica e cultural do país. Devido o número de espécies de Bauhinia utilizadas na etnofarmacologia e o aumento na investigação de produtos naturais como alternativa terapêutica, principalmente em comunidades rurais onde o acesso a 
medicação, é fator complicativo no tratamento clínico, o número de pesquisas com plantas do grupo tem se elevado nos últimos anos (Brito et al., 2020).

Além disto, é preciso destacar que o país possui uma taxa elevada de Doenças Transmitidas por Alimentos (DTA), que podem ser causadas por vírus, bactérias, parasitas e produtos químicos. Destaca-se, que a maior ocorrência de surtos de doença transmitida por alimentos foi associada à Salmonella sp. e Escherichia coli, sendo imprescindível a busca por alternativas terapêuticas no combate a esses agentes etiológicos (Brasil, 2019).

Com relação à evolução das publicações nas bases de artigos Scopus, Web of Science e The Lens (Figura 4) com os descritores selecionados, observou-se que as indexações iniciaram em 1981 ( $\mathrm{n}=1)$ e alcançaram limiar em 2016 (Scopus=12, $\mathrm{WoS}=6$ e The Lens $=43$ ). O primeiro manuscrito indexado buscou realizar um ensaio de sensibilidade antifúngica com extrato de folhas, caule e fruto da espécie Bauhinia candicans G. Bentham, encontrada na Argentina (Lorenti et al., 1981).

Figura 4. Evolução anual de artigos indexados nas bases Scopus, The Lens e Web of Science com os termos "Bauhinia AND Antimicrobial".

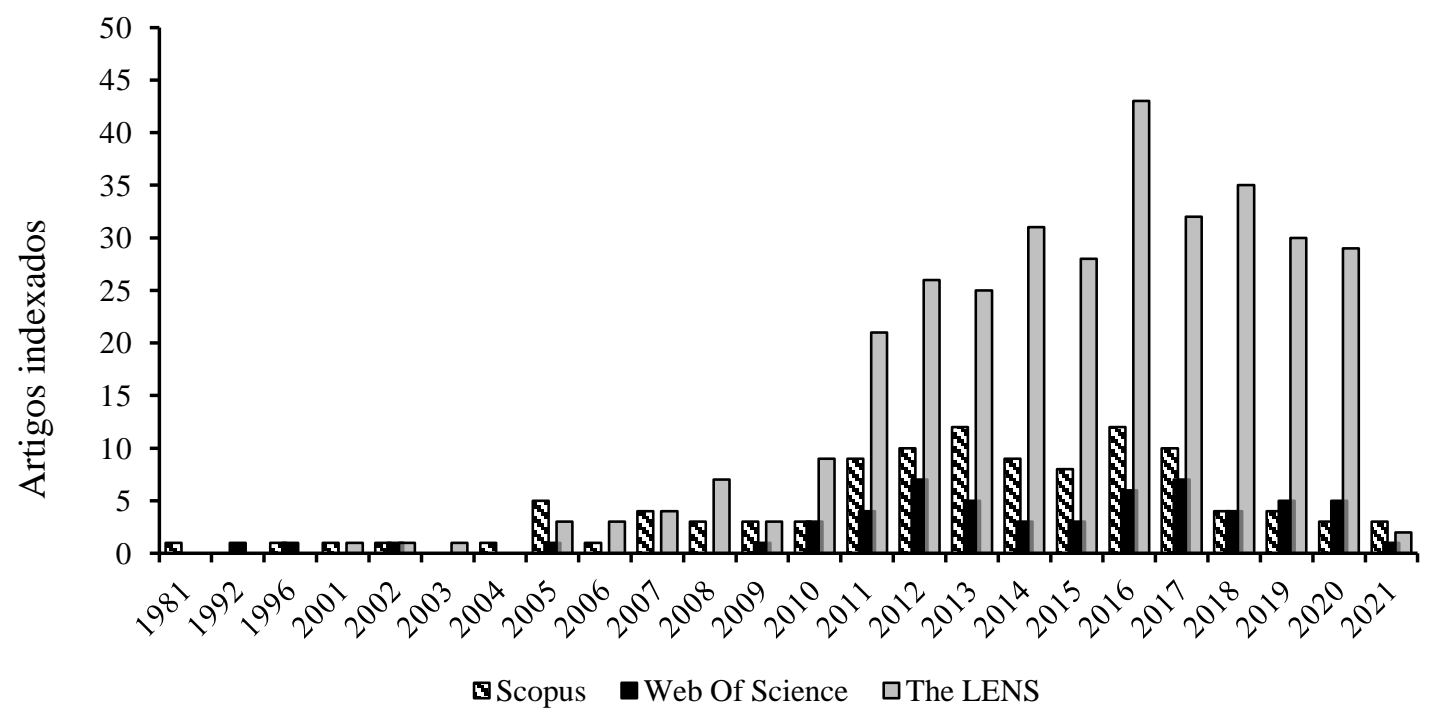

Fonte: Autores (2021).

A busca demonstrou que 13 espécies aparecem com destaque nos estudos com atividades antimicrobianas para o gênero são elas: Bauhinia forficata Link., Bauhinia galpinii N.E. Br., Bauhinia purpurea L., Bauhinia guianensis Aubl., Bauhinia ungulata L., Bauhinia acuminata Harms., Bauhinia kockiana Korth., Bauhinia recemosa Lam., Bauhinia variegata L., Bauhinia rufa (Bong.) Steud., Bauhinia tomentosa L., Bauhinia vahlii Wigth \& Arn. e Bauhinia blankeana Dunn (Tabela 2). Destaca-se que, estudos com B. variegata correspondem a 11,11\% das publicações. Dados da literatura, indicam que a presença de flavonoides pode ser caracterizada como fator de inibição do crescimento bacteriano nos testes realizados com $B$. variegata (Silveira et al., 2016). 
Tabela 2. Estudos com espécies de Bauhinia que aparecem em destaque com atividades antimicrobianas nas bases de dados a partir dos descritores selecionados.

\begin{tabular}{lcc}
\hline \multicolumn{1}{c}{ Espécie } & Atividade antimicrobiana & Referências \\
\hline Bauhinia forficata Link. & Antibacteriana & De Souza et al. (2021) \\
Bauhinia galpinii N.E. Br. & Antibacteriana e Antifúngica & Erhabor et al. (2020) \\
Bauhinia purpurea L. & Antibacteriana e Antifúngica & Vijayan, Joseph \& Mathew (2019) \\
Bauhinia guianensis Aubl. & Antifúngica & Cordeiro et al. (2019) \\
Bauhinia ungulata L. & Antibacteriana & Oliveira et al. (2019) \\
Bauhinia acuminata Harms. & Antimicrobiana & Alharbi et al. (2018) \\
Bahunia kockiana Korth. & Antibacteriana & Chew et al. (2018) \\
Bauhinia recemosa Lam. & Antifúngica & Badgujar et al. (2017) \\
Bauhinia variegata L. & Antibacteriana & Parekh, Karathia \& Chanda (2006) \\
Bauhinia rufa (Bong.) Steud. & Antibacteriana & Silveira et al. (2016) \\
Bauhinia tomentosa L. & Antibacteriana e Antifúngica & Ajani, 2016 \\
Bauhinia vahlii Wigth \& Arn. & Antibacteriana e Antifúngica & Shukla \& Gahlot, (2018) \\
Bauhinia blankeana Dunn & Antibacteriana e Antifúngica & Hu et al. (2017) \\
\hline
\end{tabular}

Fonte: Autores (2021).

De acordo com a Flora do Brasil 2020 (2021), das espécies de Bauhinia que aparecem em destaque nas bases de dados três estão listadas para o Nordeste do Brasil são elas: B. forficata, B. ungulata e B. purpurea. Na literatura, essas espécies são utilizadas popularmente na região para tratamento de doenças gastrointestinais, anti-inflamatória, redução das taxas de colesterol, triglicerídeos e principalmente no tratamento do diabetes, sendo importante aprofundar as investigações e busca de atividades e compostos antimicrobianos e confirmação de propriedades curativas de plantas do táxon (Conceição et al., 2011; Macêdo et al., 2015).

A busca por ativos antimicrobianos com espécies de Bauhinia aparecem com maior frequência relacionadas as áreas de Farmácia, Toxicologia e Farmacêutica (31,8\%), seguidas de Bioquímica, Genética e Biologia Molecular (15,1\%) e Imunologia e Microbiologia (7,8\%) (Tabela 3). O valor expressivo de trabalhos relacionados a área médica, justifica-se pelo fato de plantas do grupo estarem sendo experimentadas no tratamento alternativo, para doenças como o diabetes mellitus pelas comunidades tradicionais (Salvi et al., 2016) e indústria farmacêutica (Silva et al., 2018).

Tabela 3. Distribuição das publicações indexadas por áreas nas bases Scopus, Web of Science e The Lens.

\begin{tabular}{|c|c|}
\hline Áreas & $\%$ \\
\hline Farmacologia, Toxicologia e Farmacêutica & 31,80 \\
\hline Bioquímica, Genética e Biologia Molecular & 15,10 \\
\hline Imunologia e Microbiologia & 7,80 \\
\hline Agricultura e Ciências Biológicas & 7,30 \\
\hline Química & 6,70 \\
\hline Ciências Ambientais & 2,20 \\
\hline Engenharia Química & 1,70 \\
\hline Odontologia & 1,10 \\
\hline Outros & 9,50 \\
\hline
\end{tabular}

Fonte: Autores (2021).

Quanto ao mapeamento dos termos relevantes a partir dos descritores "Bauhinia AND Antimicrobial", verificou-se que o cluster verde agrupam-se palavras-chave que relacionam os estudos das atividades antibacterianas ("Antibacterial activity", "Escherichia coli", "Pseudomonas aeruginosa" e "Staphylococcus aureus") e antifúngicas ("Antifungal activity", “Candida albicans" e "Minimum inhibitory concentration") com plantas do táxon (Figura 5). 
Figura 5. Mapeamento dos termos relevantes com os descritores "Bauhinia AND Antimicrobial".

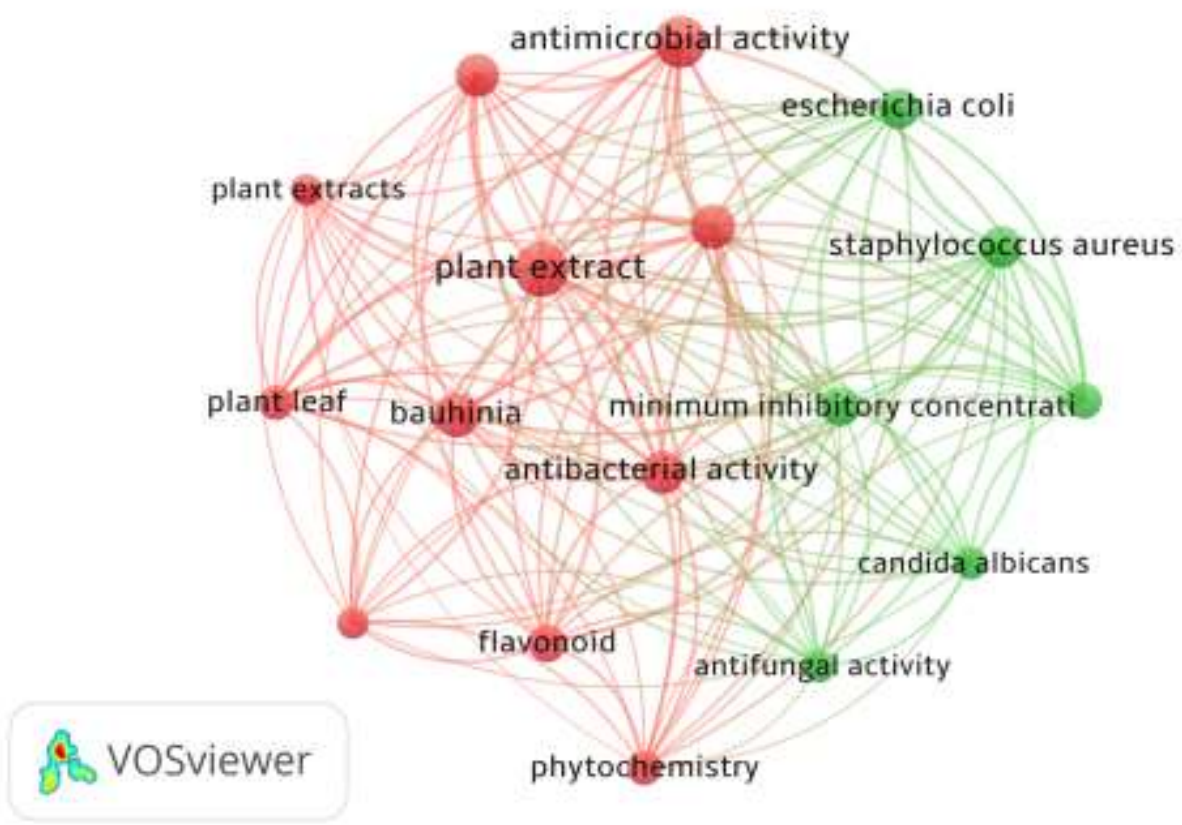

Fonte: Elaborada pelos autores deste artigo com dados da Base Scopus com o aporte do software VOSviewer para a geração do mapa (2021).

A Figura 5 mostra que o cluster em vermelho formado pelo agrupamento das palavras-chave que aparecem nos estudos estão relacionadas as atividades antimicrobianas ("Antimicrobial activity"), antioxidantes ("Antioxiant activity"), extratos vegetais de espécies de Bauhinia ("Bauhinia", "Plant extract" e "Plant leaf") e triagem de metabólitos secundários ("Flavonoid" e "Phytochemistry"). A relação entre estes termos justifica-se pela ação de compostos majoritários, como, flavonoides, taninos e polifenóis. A identificação dos compostos majoritários demostrou componentes antimicrobianos que agem em direção a patógenos fúngicos e bacterianos. A natureza anfifílica dos bioativos isolados de Bauhinia exibe atividade bacteriana através de três estágios: fixação da membrana celular, modificação fluidez do plasmalema da bactéria e ruptura da estrutura da membrana plasmática (Chew et al., 2018).

\section{Conclusão}

O mapeamento tecnológico evidenciou que na última década houve um crescimento nas indexações de tecnologias relacionadas ao táxon e suas propriedades antimicrobianas. Foi possível concluir que o maior número de patentes está associado as preparações medicinais, por exemplo medicamentos tradicionais à base de ervas. E que os Estados Unidos da América é o país com maior número de tecnologias indexadas com plantas do grupo com os descritores selecionados.

O mapeamento científico, demonstrou que a Índia vem se destacando na indexação de trabalhos científicos. O Brasil aparece em segundo lugar em número de pesquisas o que demonstra interesse e busca por propriedades terapêuticas antimicrobianas com espécies de Bauhinia, apesar disto, faz-se necessário que haja maior produção de tecnologias, fator preponderante para o desenvolvimento técnico e científico do país. As maiores concentrações de trabalhos foram em áreas com finalidade médica como Farmácia, Toxicologia e Farmacêutica, o que demonstra o interesse e busca de ativos antimicrobianos com finalidades terapêuticas.

O mapeamento dos termos mais significativos, identificou 13 palavras-chave que formam dois agrupamentos, relacionados diretamente as atividades antimicrobianas. Com os agrupamentos formados foi possível concluir, que a busca por 
compostos majoritários e sua identificação está diretamente associada aos estudos que testam as potencialidades antimicrobianas de espécies do grupo.

Como perspectiva para futuras produções, sugere-se a ampliação do mapeamento prospectivo, utilizando outras bases de artigos como Google Acadêmico e Science Direct e de patentes como European Patent Office (EPO) e United States Patent Trademarks Office (USPTO), considerando que estes estudos colaboram com a pesquisa e desenvolvimento tecnológico de produtos naturais e compostos antimicrobianos advindos de espécies do táxon.

\section{Referências}

Ajani, O. O., Owoeye, T. F., Olasehinde, G. I., Akinlabu, D. K., Owolabi, F. E., \& Audu, O. Y. (2016). Characterization, proximate composition and evaluation of antimicrobial activity of seed oil of Bauhinia tomentosa. Journal of Biological Sciences, 16(4), 102-111.

Alharbi, N. S., Govindarajan, M., Kadaikunnan, S., Khaled, J. M., Almanaa, T. N., Alyahya, S. A., \& Sudha, A. (2018). Nanosilver crystals capped with Bauhinia acuminata phytochemicals as new antimicrobials and mosquito larvicides. Journal of Trace Elements in Medicine and Biology, 50(1), 146-153.

Badgujar, N. V., Mistry, K. N., Chudasama, P. N., \& Patel, J. S. (2017). In vitro antioxidant and cytotoxic effects of methanol extracts of Vitex negundo, Lantana camara, Bauhinia variegata and Bauhinia racemosa on human cancer cell lines. Indian Journal of Pharmaceutical Sciences, 79(3), 431-437.

Brito, V. P., de Freitas, M. C., Gomes, D. C., \& de Oliveira, S. V. (2020). A fitoterapia como uma alternativa terapêutica complementar para pacientes com Diabetes Mellitus no Brasil: uma revisão sistemática. Saúde e meio ambiente: revista interdisciplinar, 9(1), 189-204.

BRASIL. (2019). Vigilância em saúde no Brasil 2003-2019. https://portalarquivos2.saude.gov.br/images/pdf/2019/setembro/25/boletim-especial-21ago19web.pdf

CDC- Centers for Diase Control and Prevetion. (2019). Threats of antibiotic resistance in the United States. http:// www.cdc.gov/DrugResistance/BiggestThreats.html

Chew, Y. L., Mahadi, A. M., Wong, K. M., \& Goh, J. K. (2018). Anti-methicillin-resistance Staphylococcus aureus (MRSA) compounds from Bauhinia kockiana Korth. And their mechanism of antibacterial activity. BMC complementary and alternative medicine, 18(1), 1-9.

Conceição, G. M., Ruggieri, A. C., Araújo, M. D. F. V., da Conceição, T. T. M. M., \& da Conceição, M. A. M. M. (2011). Plantas do cerrado: comercialização, uso e indicação terapêutica fornecida pelos raizeiros e vendedores, Teresina, Piauí. Scientia Plena, 7(12), 1-6.

Cordeiro, J. D. S., Carvalho, J. M., Feitosa, A. D. O., Pinheiro, E. A. A., Marinho, P. S. B., \& Marinho, A. M. D. R. (2019). Brefeldin A and other chemical constituents from endophytic fungus Scedosporium apiospermum. Revista Virtual de Quimica, 11(1), $210-217$.

Chechinel Filho, V. (2009). Chemical composition and biological potential of plants from the genus Bauhinia. Phytotherapy Research: An International Journal Devoted to Pharmacological and Toxicological Evaluation of Natural Product Derivatives, 23(10), 1347-1354.

De Carvalho, T. V., Nascimento, M. G. P., Bittencourt, C. B., \& de Andrade, I. M. (2020). Prospecção Científica e Tecnológica de Malpighia emarginata DC.(Malpighiaceae): espécie economicamente importante do Brasil. Cadernos de Prospecção, 13(3), 862.

Erhabor, J. O., Omokhua, A. G., Ondua, M., Abdalla, M. A., \& McGaw, L. J. (2020). Pharmacological evaluation of hydro-ethanol and hot water leaf extracts of Bauhinia galpinii (Fabaceae): A South African ethnomedicinal plant. South African Journal of Botany, 128(1), 28-34.

Farias, F. L., Pires, L. L. S., da Silva Júnior, R. I., Pavão, J. M. D. S. J., Rocha, T. J. M., \& dos Santos, A. F. (2018). Avaliação da atividade antibacteriana de extrato etanólico da Bauhinia forficata L. Diversitas Journal, 3(2), 402-411.

Fontes, A. R. (2019). Transferência de Biotecnologia. Revista Online de Pesquisa: Propriedade Intelectual, 2(2), 98-110.

Freitas, G. D., de Lima, C. P., Coelho, D. F. S., Moraes, M. O., Lima, G. L., \& Alves, W. R. (2021). Uso de diferentes métodos no controle do desenvolvimento do Staphylococcus aureus: uma revisão da literatura. Research, Society and Development, 10(2), e40310212546-e40310212546.

Gil, A. C (2008). Como elaborar projetos de pesquisa. 4. ed. São Paulo: Atlas.

Hasenclever, L., Paranhos, J., Costa, C. R., Cunha, G., \& Vieira, D. (2017). A indústria de fitoterápicos brasileira: desafios e oportunidades. Ciência \& Saúde Coletiva, 22(1), 2559-2569.

Hu, X., Yuan, S. S., Ye, X. J., \& Zou, H. S. (2017). Study on extraction and antimicrobial constituents from Bauhinia blakeana Seeds. Wuyi Science Journal, 2017(1), 1-8.

Lacerda, G. M., Monteiro, Á. B., Tintino, S. R., de Araújo Delmondes, G., Fernandes, C. N., Lemos, I. C. S., \& Kerntopf, M. R. (2017). Actividad modulador acerca de antibióticos por el extracto acuoso de las hojas de Bauhinia ungulata 1. Revista Cubana de Plantas Medicinales, 21 (3), $309-317$.

Lima, C. C., Benjamim, S. C. C., \& Santos, R. F. S. D. (2017). Mecanismo de resistência bacteriana frente aos fármacos: uma revisão. CuidArte, Enferm, $11(1), 105-113$.

Lorenti, A. S., Viale, A. A., Buschi, C. A., Gonzalez, M. D., Schteingart, C. D., Iribarren, A. M., \& Pomilio, A. B. (1981). Antimicrobial activity of some Argentine higher plants. Fitoterapia, 52(2), 81-85. 
Macêdo, D. G., Ribeiro, D. A., Coutinho, H. D., Menezes, I. R., \& SOUZA, M. M. (2015). Práticas terapêuticas tradicionais: uso e conhecimento de plantas do cerrado no estado de Pernambuco (Nordeste do Brasil). Boletín Latinoamericano y del Caribe de Plantas Medicinales y Aromáticas, 14(6), 491-508.

Medeiros, S. R., de Melo Filho, A. A., da Costa, H. N., dos Santos Silva, F., dos Santos, R. C., Takahashi, J. A., \& da Silva Paulino, F. (2016). Chemical profile, antimicrobial activity, toxicity on Artemia salina and anti-acetylcholinesterase enzyme essential oil from Bauhinia ungulata L. (Fabaceae) leaves. Journal of Medicinal Plants Research, 10(29), 442-449.

Nadvorny, A., Figueiredo, D. M. S., \& Schmidt, V. (2004). Ocorrência de Salmonella sp. em surtos de doenças transmitidas por alimentos no Rio Grande do Sul em 2000. Acta Scientiae Veterinariae, 32(1), 47-51.

Oliveira, R. R., Yaochite, J. N. U., Braga, M. A., Sasahara, G. L., da Cruz Fonseca, S. G., de Vasconcelos Araújo, T. D., \& Nagao-Dias, A. T. (2019). Antioxidant and anti-inflammatory activities of Bauhinia ungulata L. (Fabaceae) on LPS-stimulated RAW 264.7 Cells. Pharmacognosy Journal, 11(1), 20-28.

Oliveira, R. R., Yaochite, J. N. U., Sasahara, G. L., Albuquerque, A. A., da Cruz Fonseca, S. G., de Vasconcelos Araújo, T. D., \& Nagao-Dias, A. T. (2020). Antioxidant, anti-inflammatory and healing potential of ethyl acetate fraction of Bauhinia ungulata L. (Fabaceae) on in vitro and in vivo wound model. Molecular biology reports, 47(4), 2845-2859.

Palludeto, A. W. A., \& Felipini, A. R. (2019). Panorama da literatura sobre a financeirização (1992-2017): uma abordagem bibliométrica. Economia $e$ sociedade, 28(1), 313-337.

Paula, C. S., Canteli, V. C. D., Hirota, B. C. K., Campos, R., de Oliveira, V. B., Kalegari, M., \& Miguel, M. D. (2014). Potencial antioxidante in vitro das folhas da Bauhinia ungulata L. Revista de Ciências Farmacêuticas Básica e Aplicada, 35(2), 15-29.

Pereira, A. C. S., Ribeiro, G. E., Souza, L. C. R., Rufino, L. R. A., Cabral, I. S. R., Boriollo, M. F. G., \& Fiorini, J. E. (2014). Atividade biológica do extrato hidroalcoólico de Bauhinia forficata Link sobre Herpetomonas samuelpessoai (Galvão.) Roitman. Revista Brasileira de Plantas Medicinais, 16(1), 585-592.

Radaelli, V., \& Paranhos, J. (2015). Caracterização da trajetória de desenvolvimento da indústria farmacêutica na Índia: elementos para aprendizado. Parcerias Estratégicas, 18(36), 09-32.

Salvi, L. C., Bersch, B., Rempel, C., \& Strohschoen, A. A. G. (2016). Percepção de indivíduos com Diabetes Mellitus sobre a utilização de plantas medicinais hipoglicemiantes. Revista Contexto \& Saúde, 16(30), 55-63.

Serafini, M. R., Quintans, J. D. S. S., Antoniolli, Â. R., dos Santos, M. R. V., \& Quintans-Junior, L. J. (2012). Mapeamento de tecnologias patenteáveis com o uso da hecogenina. Revista Geintec-Gestao Inovacao e Tecnologias, 2(5), 427-435.

Shukla, D., \& Gahlot, K. (2018). Studies on antimicrobial potential of Bauhinia Vahlii Stem extracts. Journal of Drug Delivery and Therapeutics, 8(3), 106108 .

Silva, E. L. P., Soares, J. C. F., Machado, M. J., Reis, I. M. A., \& Cova, S. C. (2020). Avaliação do perfil de produção de fitoterápicos para o tratamento de ansiedade e depressão pelas indústrias farmacêuticas brasileiras. Brazilian Journal of Development, 6(1), 3119-3135.

Silva, F. G. O., Araújo, A. D., da Silva Almeida, J. R. G., da Silva, M. V., \& dos Santos Correia, M. T. (2020). Prospecção Tecnológica de Spondias tuberosa (Anacardiaceae). Revista Geintec-Gestao Inovacao e Tecnologias, 10(3), 5546-5552.

Silva, N. C. S., Vítor, A. M., da Silva Bessa, H. H., \& Barros, R. M. S. (2017). A utilização de plantas medicinais e fitoterápicos em prol da saúde. Única cadernos acadêmicos, 3(1)1-5.

Silva, T. G. (2018). Medicamentos Fitoterápicos: Uma nova alternativa no tratamento das doenças negligenciadas. Open Journal of Aging Research, 1(5), 1-8.

Silveira, D., Faitanin, R. D., Gomes, S. M., Fagg, C. W., Fonseca-Bazzo, Y. M., Magalhães, P. O., \& Jamal, C. M. (2016). Thrombolytic Activity Evaluation Of Extracts From Fabaceae Species Bauhinia Variegata, B. Rufa, and Stryphnodendron Adstringens. PharmacologyOnline, 3(1), 1-5.

Sousa, J. N., de Oliveira, A. B. M., Ferreira, A. K., Silva, E., de Sousa, L. M. S., França Rocha, M. C., \& Medeiros Barreto, H. (2021). Modulation of the resistance to norfloxacin in Staphylococcus aureus by Bauhinia forficata link. Natural product research, 35(4), 681-685.

Souza, L. F.P., Martins, C. P. T., Martins, C. F., Caires, P. T. P. R. C., Aragão, V. S., Aragão, O. S., \& Chaves, M. C. (2021). Fatores de risco e mortalidade em pacientes criticamente enfermos com infecções por microrganismos multirresistentes. Revista Eletrônica Acervo Saúde, 13(4), e7319-e7319.

Flora do Brasil 2020 (2021). Bauhinia in Flora do Brasil 2020. http://floradobrasil.jbrj.gov.br/reflora/floradobrasil/FB22811

Van Eck, N. J., \& Waltman, L. (2010). Software survey: VOSviewer, a computer program for bibliometric mapping. Scientometrics, 84(2), 523-538.

Vijayan, R., Joseph, S., \& Mathew, B. (2019). Anticancer, antimicrobial, antioxidant, and catalytic activities of green-synthesized silver and gold nanoparticles using Bauhinia purpurea leaf extract. Bioprocess and biosystems engineering, 42(2), 305-319.

WIPO- World Intellectual Property Organizacion. (2021). International Patent Classification (IPC). https://patentscope.wipo.int/search/pt/struct uredSearch.jsf. 